\title{
Influence of corneal power on intraocular lens power of the second eye in the SRK/T formula in bilateral cataract surgery
}

\author{
Young Choi ${ }^{1}$, Youngsub Eom ${ }^{1,2^{*}} \mathbb{D}$, Jong Suk Song ${ }^{1}$ and Hyo Myung Kim ${ }^{1}$
}

\begin{abstract}
s
Background: To evaluate the effect of different adjustments of the refractive outcome of the first eye according to corneal power $(K)$ in order to improve the intraocular lens (IOL) power calculation of the second eye in the SRK $T$ formula.

Methods: One hundred thirty-four patients who underwent uncomplicated bilateral, sequential phacoemulsification with AcrySof IQ implantation were enrolled. The optimal partial adjustment of the refractive outcome of the first eye according to $\mathrm{K}$ was retrospectively analyzed using a regression formula.

Results: In all patients, the optimal partial adjustment of the refractive outcome of the first eye was calculated as 56\%. For $\mathrm{K}$ values between 42.8 D and 44.6 D, the optimal partial adjustment was calculated as 30\%; however, this adjustment of the first eye did not significantly improve the refractive outcome in the second eye of the subgroup with $\mathrm{K}$ values between $42.8 \mathrm{D}$ and $44.6 \mathrm{D}$. For $\mathrm{K}$ values greater than $44.6 \mathrm{D}$ or less than $42.8 \mathrm{D}$, the optimal partial adjustments were calculated as $69 \%$ and $81 \%$, respectively. According to these results, the adjustment of the first eye significantly improved the refractive outcome in the second eye from 0.36 to $0.26 \mathrm{D}(P<0.001)$ in the entire data set. This result was significantly lower than that using a single partial adjustment (56\%) (0.28 D; $P=0.027)$.

Conclusions: For $K$ values greater than $44.6 \mathrm{D}$ or less than $42.8 \mathrm{D}$, an approximately $70-80 \%$ adjustment of the first eye error should be considered. In contrast, for $\mathrm{K}$ values between 42.8 D and 44.6 D, a 30\% or less adjustment should be considered in the SRK/T formula.
\end{abstract}

Keywords: Bilateral cataract extraction, Corneal power, Intraocular lens power, SRK/T formula

\section{Background}

Postoperative vision after cataract surgery has been greatly improved by advances in surgical techniques, precise biometry techniques, and intraocular lens (IOL) power calculation formulas [1-7]; however, the refractive error is still a major concern in cataract surgery.

A previous study demonstrated that using corneal power (K)-specific constants improved the refractive outcomes predicted by the Sanders-Retzlaff-Kraff (SRK)/ $\mathrm{T}$ formula because it predicts a myopic refractive error for a steep cornea and a hyperopic refractive error for a flat cornea [4]. In that study, the refractive error showed

\footnotetext{
* Correspondence: hippotate@hanmail.net
${ }^{1}$ Department of Ophthalmology, Korea University College of Medicine, Seoul,

* Correspondence: hippotate@hanmail.net
${ }^{1}$ Department of Ophthalmology, Korea University College of Medicine, Seoul, South Korea

${ }^{2}$ Department of Ophthalmology, Ansan Hospital, Korea University College of Medicine, 123, Jeokgeum-ro, Danwon-gu, Ansan-si, Gyeonggi-do 15355, Medicine, 123,
South Korea
}

a distribution between $0.25 \mathrm{D}$ to $-1.00 \mathrm{D}$ for $\mathrm{K}$ values prove the IOL calculation for the second eye due to the symmetry between the two eyes [8-10]. Thus, if the first eye shows a $-1.00 \mathrm{D}$ myopic shift with a $\mathrm{K}$ of $47 \mathrm{D}$, the second eye is likely to show a similar myopic shift, which is different from the average refractive error. Therefore, we hypothesized that increasing the magnitude of the wirst eye error for a steep or flat corwould improve the refractive outcome in the second designed to evaluate the effect of different adjustments of the refractive outcome of the first eye according to $\mathrm{K}$ for improving the IOL power calculation of the second eye in the SRK/T formula. cataract surgery, previous studies have shown that the the data made avalable in this anticle, unless otherwise stated. 


\section{Methods}

\section{Study population}

This retrospective cross-sectional study included 268 eyes from 134 patients who underwent uncomplicated bilateral, sequential phacoemulsification with IOL implantation at Korea University Anam Hospital, Seoul, Korea between April 2008 and December 2015. An AcrySof IQ (SN60WF, Alcon, Fort Worth, TX, USA) IOL was implanted in both eyes of each patient. Patients who had best corrected visual acuities (BCVA) better than or equal to $20 / 40$ in both eyes after cataract surgery were included. Patients with a traumatic cataract, prior ocular surgery (such as penetrating keratoplasty or refractive surgery), complicated surgery (such as posterior capsule rupture), or postoperative complications were excluded. Institutional Review Board (IRB) approval was obtained from Korea University Anam Hospital, Seoul, Korea for this study. All research and data collection methods followed the tenets of the Helsinki agreement. The data used in this study were de-identified for the sake of privacy for subjects.

\section{Patient examination}

All measurements were taken by a trained ophthalmic examiner who measured the preoperative axial length (AL) and $\mathrm{K}$ with optical biometry using an IOLMaster version 5.02 or higher (Carl Zeiss Meditech, Jena, Germany). IOL power was calculated using the SRK/T formula of the IOLMaster. The data-adjusted A-constant for AcrySof IQ was 119.0, calculated in our previous study using the Haigis constant optimization Excel spreadsheet (Microsoft Inc., Redmond, WA, USA) for optical biometry $[4,11]$.

Postoperative uncorrected visual acuity (UCVA), manifest refraction, and BCVA were measured at postoperative visits between three and 10 weeks.

\section{Surgical technique}

Phacoemulsification and IOL implantation were performed under topical anesthesia with $0.5 \%$ proparacaine hydrochloride (Alcaine; Alcon, Fort Worth, TX, USA) via a 2.2 or $2.75 \mathrm{~mm}$ temporal clear corneal incision by an experienced surgeon (HM.K.). The IOL was inserted into the capsular bag.

\section{Main outcome measure(s)}

The refractive error was defined as the difference between the observed refractive spherical equivalent three to 10 weeks postoperatively and the predicted refraction (spherical equivalent) by IOLMaster using the SRK/T formula (refractive error = postoperative spherical equivalent - preoperative predicted refraction). The mean absolute refractive error (MAE) was defined as the mean absolute value of the refractive error.
The optimal partial adjustment of the refractive error of the first eye according to $\mathrm{K}$ for improving the IOL calculation of the second eye was analyzed in retrospect using the corrective regression formula [9]: $R \varkappa_{\text {cor }}=R \varkappa_{\exp }+\beta \mathrm{x}$ $P \varkappa_{\text {err }}$, where $R \varkappa_{\text {cor }}$ is the observed refractive spherical equivalent of the second eye, $R \varkappa_{\exp }$ is the expected refractive prediction of the second eye, $P \mathcal{x}_{\mathrm{err}}$ is the refractive error of the first eye, and $\beta$ is a correlation coefficient that is a magnitude of adjustment of first-eye error for improving the refractive outcome of the second eye.

The adjusted MAE of the second eye without considering $\mathrm{K}\left(\mathrm{MAE}_{\mathrm{WCP}}\right)$ was defined as the MAE of the second eye using a partial adjustment for the refractive error of the first eye with a calculated correlation coefficient from the entire data set by the corrective regression formula. The adjusted MAE of the second eye according to $\mathrm{K}\left(\mathrm{MAE}_{\mathrm{ACP}}\right)$ was defined as the MAE of the second eye using a partial adjustment for the refractive error of the first eye with calculated correlation coefficients for within cut-off and outside cut-off values. To decide the $\mathrm{K}$ cut-off value, the correlation coefficient for the partial adjustment was calculated from the cumulative subgroups based on K. Each cumulative subgroup was made according to both an increase in $\mathrm{K}$ from 42 to $47 \mathrm{D}$ and a decrease in $\mathrm{K}$ from 47 to $42 \mathrm{D}$ at $0.2 \mathrm{D}$ intervals. After that, the lower and upper cut-off $\mathrm{K}$ values, which showed a deviation from the correlation coefficient increasing or decreasing trend, were decided.

\section{Statistical analysis}

Descriptive statistics were obtained using the Statistical Package for the Social Sciences (SPSS) version 21.0 (IBM Corp., Armonk, NY, USA). The KolmogorovSmirnov test was performed to assess data distribution normality. Paired $t$-tests were used for parametric continuous variables and the Wilcoxon signed rank test was used for nonparametric continuous variables according to the results of normality distribution tests. Repeatedmeasures analysis of variance (ANOVA) with the Bonferroni correction were performed to assess statistical differences among the unadjusted $\mathrm{MAE}, \mathrm{MAE}_{\mathrm{WCB}}$ and $\mathrm{MAE}_{\mathrm{ACP}}$. Results were considered statistically significant if the $p$-value was less than 0.05 . A post-hoc power analysis using the Wilcoxon signed-rank test option of G*power, version 3.1.9.2 (Franz Paul, Kiel, Germany), was conducted to determine study power.

\section{Results}

One hundred thirty-four patients were included in this study. Of the 134 patients, 50 (37.3\%) were men and 84 were women. The mean age $( \pm \mathrm{SD})$ was $68.6 \pm 8.5$ years (range, 43 to 90 years). The mean K, AL, calculated IOL power, preoperative predicted refraction, postoperative refraction, and refractive error are shown in Table 1. 
Table 1 Clinical characteristics of patients and eyes included in the present study $(n=134)$

\begin{tabular}{lccll}
\hline Parameter & Patients & First eye & $\begin{array}{l}\text { Second } \\
\text { eye }\end{array}$ & $P^{\text {value }}$ \\
\hline Age, years (SD) & $68.6(8.5)$ & & & - \\
Sex (Male:Female) & $50(37.3)$ : & & & - \\
(\%) & $84(62.7)$ & & & \\
Corneal power, & & $44.22(1.43)$ & $44.20(1.47)$ & 0.687 \\
D (SD) & & & \\
Axial length, mm (SD) & $23.55(0.95)$ & $23.52(0.89)$ & $0.691^{\mathrm{b}}$ \\
IOL power, D (SD) & $20.8(2.7)$ & $20.9(2.4)$ & $0.395^{\mathrm{b}}$ \\
$\begin{array}{l}\text { Predicted refraction, } \\
\text { D (SD) }\end{array}$ & $-0.26(0.24)$ & $-0.26(0.23)$ & 0.847 \\
Refraction at postop 3 & & $-0.22(0.55)$ & $-0.21(0.50)$ & $0.884^{\mathrm{b}}$ \\
to 10 weeks, D (SD) & & & \\
Refractive error, & $0.04(0.49)$ & $0.04(0.45)$ & 0.990 \\
D (SD) & & & &
\end{tabular}

Data are mean (SD) except for parameter sex, which are $n$ (\%)

$S D$ standard deviation, $D$ diopters, $O L$ intraocular lens

${ }^{a}$ Paired $t$-test was used for parametric continuous variables

${ }^{b}$ Wilcoxon signed rank test was used for nonparametric continuous variables

There was a significant correlation in $\mathrm{K}\left(\mathrm{R}^{2}=0.915, P\right.$ $<0.001), \mathrm{AL}\left(\mathrm{R}^{2}=0.912, \mathrm{P}<0.001\right)$, and IOL power $\left(\mathrm{R}^{2}\right.$ $=0.883, \mathrm{P}<0.001)$ between the first and second eyes. There was also a significant correlation between the refractive error of the first and second eyes $\left(\mathrm{R}^{2}=0.366, \mathrm{P}\right.$ $<0.001$; Fig. 1). According to the corrective regression formula $^{6}$, the correlation coefficient $(\beta)$ was 0.56 in all patients using the SRK/T formula. This means that the

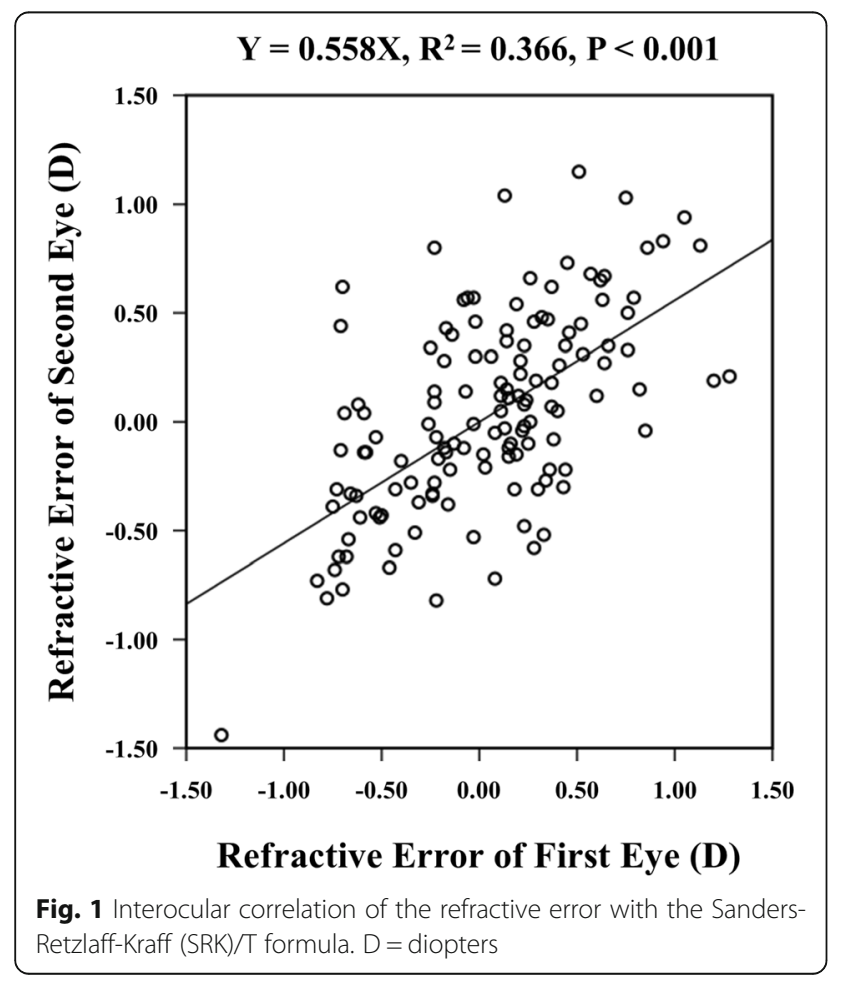

optimal partial adjustment of the refractive error of the first eye for IOL calculation of the second eye was determined to be $56 \%$. According to these results, the MAE of second eyes decreased from 0.36 to $0.28 \mathrm{D}(P<0.001$; Table 2).

There was a very weak positive correlation between $\mathrm{AL}$ and refractive error $\left(\mathrm{R}^{2}=0.025, P=0.010\right.$; Fig. 2a). On the other hand, a negative correlation was observed between $K$ and refractive error $\left(R^{2}=0.140, P<0.001\right.$; Fig. 2b). According to the regression equation, the refractive error could be zero when $\mathrm{K}$ was $44.43 \mathrm{D}$. As $\mathrm{K}$ increased, the refractive error showed a tendency for myopic refractive outcomes. On the contrary, as $\mathrm{K}$ decreased, the refractive error showed a tendency for hyperopic refractive outcomes.

Figure 3 shows the correlation coefficients of each cumulative subgroup which were calculated according to both an increase in $\mathrm{K}$ from $42 \mathrm{D}$ (heavy line) and a decrease in $\mathrm{K}$ from $47 \mathrm{D}$ (light line). The correlation coefficients of the cumulative subgroups tended to decrease as $\mathrm{K}$ increased from $42 \mathrm{D}$ and tended to decrease as $\mathrm{K}$ decreased from $47 \mathrm{D}$. The lower and upper cut-off K values were determined to be 42.8 and $44.6 \mathrm{D}$, respectively. The correlation coefficient for values less than the lower cut-off $(\mathrm{K}<42.8 \mathrm{D})$ was calculated as 0.81 and that for values over the upper cut-off $(44.6 \mathrm{D} \leq \mathrm{K})$ was calculated as 0.69 . The lowest correlation coefficient was observed between 42.8 and 44.6 D (correlation coefficient $=0.30)$.

The $\mathrm{MAE}_{\mathrm{ACP}}( \pm \mathrm{SD})(0.26 \pm 0.23 \mathrm{D})$ was smaller than the $\operatorname{MAE}_{\mathrm{WCP}}(0.28 \pm 0.22 \mathrm{D})$ in the entire dataset $(P=0.027$; Table 2 and Fig. 4). In a subgroup analysis, neither the $\mathrm{MAE}_{\mathrm{WCP}}$ nor $\mathrm{MAE}_{\mathrm{ACP}}$ of the subgroup within the cut-off values improved the refractive outcome in the second eye. Otherwise, both the $M A E_{W C P}$ and $M_{A A} E_{A C P}$ of the subgroup outside the cut-off values significantly improved the refractive outcome from $0.40 \mathrm{D}$ to $0.24 \mathrm{D}$ and $0.21 \mathrm{D}$, respectively $(P<0.001, \quad \mathrm{P}<0.001$, respectively $)$ The $\operatorname{MAE}_{\mathrm{ACP}}(0.21 \pm 0.21 \mathrm{D})$ was significantly smaller than the $\operatorname{MAE}_{\mathrm{WCP}}(0.24 \pm 0.20 \mathrm{D})$ in the subgroup outside of the cut-off values $(P=0.032)$.

The $\mathrm{MAE}_{\mathrm{ACP}}$ and $\mathrm{MAE}_{\mathrm{WCP}}$ in the subgroup outside of the cut-off values were used in a post-hoc power analysis. The correlation between the $\mathrm{MAE}_{\mathrm{ACP}}$ and $\mathrm{MAE}_{\mathrm{WCP}}$ was 0.925 and the effect size was 0.375 . The effect size of 0.375 and a two-tailed alpha of 0.05 in the subgroup analysis with 70 patients led to a power of 0.86 .

\section{Discussion}

A high degree of interocular symmetry of biometry between the two eyes is helpful in IOL power calculation for the second eye in bilateral sequential cataract surgery [12]. Most patients in this study showed strong interocular correlation with $\mathrm{K}\left(\mathrm{R}^{2}=0.915\right), \mathrm{AL}\left(\mathrm{R}^{2}=0.912\right)$, and 
Table 2 Comparison of the unadjusted mean absolute refractive error (MAE $E_{U N A D}$ ), adjusted MAE without considering corneal power $\left(M A E_{W C P}\right)$, and adjusted MAE according to corneal power $\left(M_{A} E_{A C P}\right)$ of the second eye in each subgroup (Repeated measures ANOVA with Bonferroni correction)

\begin{tabular}{|c|c|c|c|c|}
\hline \multicolumn{2}{|c|}{ MAE of second eye (D) } & Total $(n=134)$ & $\begin{array}{l}\text { Within cut-off values [line break] } 42.8 \mathrm{D} \leq \\
\mathrm{K}<44.6 \mathrm{D}(n=64)\end{array}$ & $\begin{array}{l}\text { Outside cut-off values [line break] } \mathrm{K}<42.8 \mathrm{D} \\
\text { or } 44.6 \mathrm{D} \leq \mathrm{K}(n=70)\end{array}$ \\
\hline \multicolumn{2}{|c|}{ Unadjusted MAE (MAE UNADJ), D (SD) } & $0.36(0.27)$ & $0.33(0.26)$ & $0.40(0.28)$ \\
\hline \multicolumn{2}{|c|}{$\begin{array}{l}\text { Adjusted MAE without considering corneal } \\
\text { power }\left(M A E_{W C P}\right), D(S D)\end{array}$} & $0.28(0.22)$ & $0.33(0.24)$ & $0.24(0.20)$ \\
\hline \multicolumn{2}{|c|}{$\begin{array}{l}\text { Adjusted MAE according to corneal power } \\
\left(M A E_{A C P}\right), D(S D)\end{array}$} & $0.26(0.23)$ & $0.31(0.24)$ & $0.21(0.21)$ \\
\hline \multirow[t]{3}{*}{$P$ value } & $M_{\text {ANAD }}$ VS. MAE & $<0.001$ & $>0.999$ & $<0.001$ \\
\hline & MAE UNADJ VS. MAE & $<0.001$ & $>0.999$ & $<0.001$ \\
\hline & $M A E_{W C P}$ Vs. MAE & 0.027 & 0.549 & 0.032 \\
\hline
\end{tabular}

$D$ diopters, MAE mean absolute refractive error, $K$ mean corneal power, $S D$ standard deviation

IOL power $\left(R^{2}=0.883\right)$. Similarly, Covert et al., [8] showed strong interocular correlations with $\mathrm{K}\left(\mathrm{R}^{2}=0.88\right)$ and $\mathrm{AL}$ measurements $\left(R^{2}=0.96\right)$ and several other studies have shown a high degree of interocular correlation with $\mathrm{K}$, anterior chamber depth, and AL measurements $[10,12,13]$. Actually, refractive outcome of the second eye was improved using the refractive error observed in the first eye [8-10].

This study evaluated the effect of different adjustments of the refractive error observed in the first eye according to $\mathrm{K}$ on the refractive outcome of the second eye using the SRK/T formula. The results showed that the method of different adjustments according to $\mathrm{K}$ value significantly improved the refractive outcome of the second eye compared to the method of fixed partial adjustments. When corneal power was not considered, the optimal partial adjustment of the refractive error of the first eye was calculated to be $56 \%$ in the entire dataset. The results of the present study using the SRK/T formula were similar to the previous studies. Covert et al. [8] performed a study demonstrating the effectiveness of a partial adjustment (50\%) to the refractive error observed in the first eye for IOL power calculation of the second eye to improve the refractive outcome of the second eye using the Holladay I and SRK II formulas. Olsen [9] demonstrated similar results using the SRK II (56\%), SRK/T (38\%), and more recent Olsen formulas (27\%). Otherwise, there was no benefit to full adjustment of the refractive error of the first eye $[8,13]$.

Olsen [9] showed that the magnitude of the partial adjustment of refractive outcome of the first eye and the improvement in refractive outcome of the second eye differ depending on the IOL power calculation formula. When the formula was less accurate, more adjustments were needed, and greater benefits were shown after the correction. In the present study, the correlation coefficients of each cumulative subgroup were calculated to determine the cut-off values according to $\mathrm{K}$ and the cut-
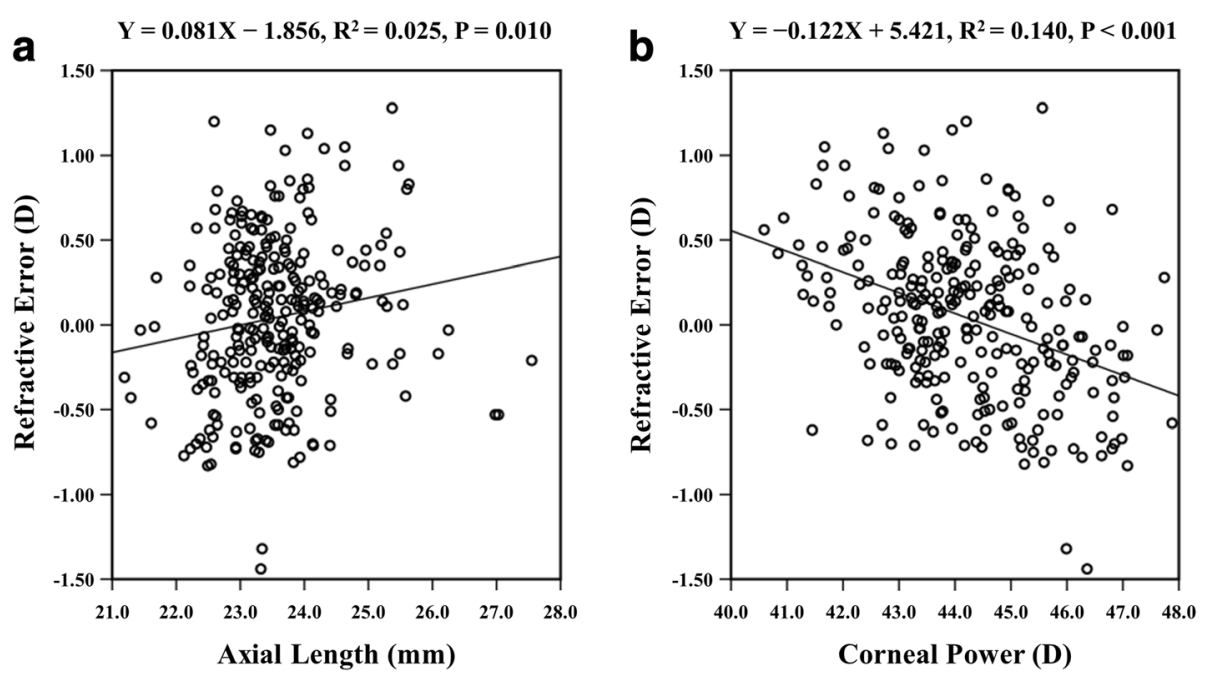

Fig. 2 Comparison of axial length, corneal power, and refractive error with the Sanders-Retzlaff-Kraff (SRK)/ $T$ formula in both eyes $(n=268)$. a Relation between axial length and refractive error. $\mathbf{b}$ Relation between corneal power and refractive error. $\mathrm{D}=$ diopters 


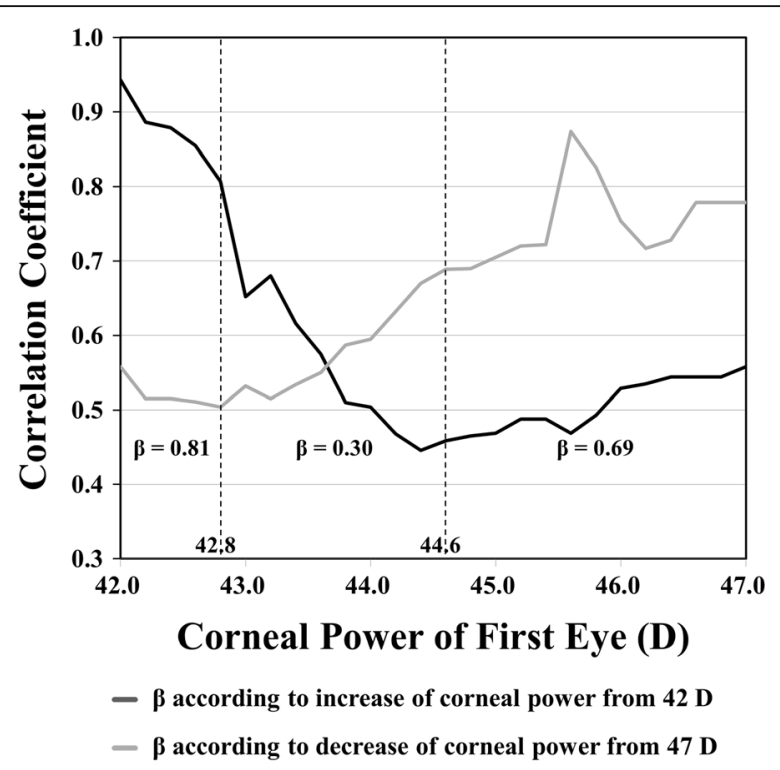

Fig. 3 Graph showing the correlation coefficient according to corneal power. The correlation coefficients ( $Y$-axis) of each cumulative subgroup (X-axis), which contained subjects whose corneal power was less than the corneal power on the X-axis, are shown as a graph according to the increase of corneal power from $42 \mathrm{D}$ (heavy line). The correlation coefficient of each cumulative subgroup, which contained subjects whose corneal power was greater than or equal to the corneal power on the X-axis, are shown as a graph according to the decrease of corneal power from $47 \mathrm{D}$ (light line). $\beta=$ Correlation coefficient

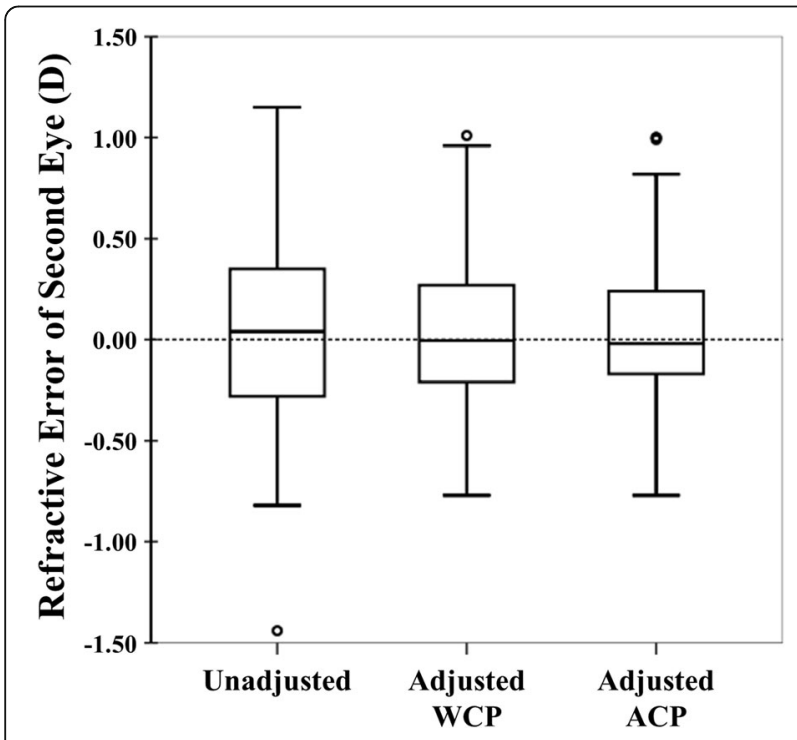

Fig. 4 The refractive error of the second eye was calculated using unadjustment, partial adjustment without considering corneal power (WCP), and partial adjustment according to corneal power (ACP) of the refractive error of the first eye using the Sanders-Retzlaff-Kraff $(\mathrm{SRK}) / T$ formula. $\mathrm{D}=$ diopters off values were set at $42.8 \mathrm{D}$ and $44.6 \mathrm{D}$. When the cornea was steeper than the upper cut-off value or flatter than the lower cut-off value, the magnitude of optimal partial adjustment of the first eye refractive error $(69 \%$, $81 \%$, respectively) were larger than that of the whole period (56\%). The lowest magnitude of optimal partial adjustment (30\%) was observed within the cut-off values. There was no benefit to partial adjustment for first eye error within the cut-off values. Otherwise, there was an improvement in the refractive outcome in the subgroups outside of the cut-off values. The $\mathrm{MAE}_{\mathrm{ACP}}$ showed a greater effect than the $M A E_{W C P}$ in the subgroup outside of the cut-off values. Thus, the magnitude of the partial adjustment of the first eye refractive error and the improvement in refractive outcome of the second eye could differ depending on the $\mathrm{K}$ in the SRK/T formula.

Sheard et al., [14] demonstrated that the SRK/T formula has non-physiologic behavior in the corrected $\mathrm{AL}$ and corneal height calculation. According to the non-physiologic behavior in the corneal height calculation, [14] the predicted corneal height tends to be overestimated as $\mathrm{K}$ increases and tends to be underestimated as $\mathrm{K}$ decreases in the SRK/T formula. Our previous study [4] and the present study demonstrated a negative correlation between $\mathrm{K}$ and the refractive error using the $\mathrm{SRK} / \mathrm{T}$ formula. In the present study, the refractive error was smallest when $\mathrm{K}$ was $44.43 \mathrm{D}$. The refractive outcome became more myopic as $\mathrm{K}$ increased and became more hyperopic as $\mathrm{K}$ decreased. These findings were similar to those noted above [14]. These results imply that the accuracy of the SRK/T formula decreases when the cornea becomes steeper or flatter. Therefore, the magnitude of adjustment of the first eye outcome should be changed according to $\mathrm{K}$ in the SRK/T formula.

There are some limitations in the present study. First, the sample size was relatively small and medical records were retrospectively reviewed. Second, UCVA, manifest refraction, and BCVA were measured at postoperative visits between three and 10 weeks, due to the retrospective nature of this study [6]. However, a previous study showed that the changes in effective lens position and refractive error of the in-the-bag AcrySof IOL were insignificant from 1 week to 6 months after surgery [15]. Third, there were a few patients who had severe differences in $\mathrm{K}$ between both eyes, and the differences in $\mathrm{K}$ were not considered in the present study. Therefore, a study on the optimal partial adjustment of the refractive error of the first eye according to $\mathrm{K}$ with a large number of patients will be necessary.

\section{Conclusions}

Partial adjustment of the refractive error of the first eye according to the regression formula improved the refractive outcome in the second eye. When the cornea is steep or flat, an approximately $70-80 \%$ magnitude 
adjustment of the first eye error should be considered in the SRK/T formula. On the contrary, when $\mathrm{K}$ is within the range of cut-off values, a magnitude adjustment of $30 \%$ or less should be considered.

\section{Abbreviations}

AL: Axial length; BCVA: Best corrected visual acuity; IOL: Intraocular lens; $\mathrm{K}$ : Corneal power; MAE: Mean absolute refractive error; MAE $\mathrm{ACP}$ : Adjusted MAE of the second eye according to K; MAE wCP: Adjusted MAE of the second eye without considering K; SRK/T: Sanders-Retzlaff-Kraff $/ \mathrm{T}$; UCVA: Uncorrected visual acuity; $\beta$ : Correlation coefficient

\section{Acknowledgements}

None

\section{Funding}

This paper was supported by Bumsuk Academic Research Fund in 2017. The funding source had no role in the design or conduct of this research.

\section{Availability of data and materials}

The datasets collected during and/or analyzed during the current study are available from the corresponding author on reasonable request.

\section{Authors' contributions}

Y.C. has made substantial contributions to acquisition of data and been involved in drafting the manuscript. Y.E. has made substantial contributions to conception and design, acquisition of data, and analysis and interpretation of data, been involved in drafting the manuscript and revising it, and given final approval of the version to be published. JS.S. and HM.K have made substantial contributions to analysis and interpretation of data and given final approval of the version to be published. All authors have read and approved the final version of this manuscript.

\section{Ethics approval and consent to participate}

This retrospective study was performed in adherence to the tenets of the Declaration of Helsinki and was approved by the IRB of Korea University Anam Hospital (IRB number: 2017AN0362). According to the IRB standard operating procedures on retrospective single center clinical study Ethics Committee of the Korea University Anam Hospital ruled that subject consent was not required for this study.

\section{Consent for publication}

Not applicable (no identifying patient data).

\section{Competing interests}

The authors declare that they have no competing interests.

\section{Publisher's Note}

Springer Nature remains neutral with regard to jurisdictional claims in published maps and institutional affiliations.

Received: 30 June 2017 Accepted: 14 December 2017

Published online: 28 December 2017

\section{References}

1. Mamalis N. Intraocular lens power accuracy: how are we doing? J Cataract Refract Surg. 2003;29(1):1-3.

2. Olsen T. Improved accuracy of intraocular lens power calculation with the Zeiss IOLMaster. Acta Ophthalmol Scand. 2007;85(1):84-7.

3. Olsen T. Calculation of intraocular lens power: a review. Acta Ophthalmol Scand. 2007;85(5):472-85.

4. Eom Y, Kang SY, Song JS, Kim HM. Use of corneal power-specific constants to improve the accuracy of the SRK $/ \mathrm{T}$ formula. Ophthalmology. 2013;120(3):477-81

5. Eom Y, Hwang HS, Hwang JY, Song JS, Kim HM. Posterior vault distance of Ciliary Sulcus-implanted three-piece intraocular lenses according to Ciliary Sulcus diameter. Am J Ophthalmol. 2017:175:52-9.

6. Eom Y, Song JS, Kim HM. Modified Haigis formula effective lens position equation for Ciliary Sulcus-implanted intraocular lenses. Am J Ophthalmol. 2016;161:142-9. e141-142
7. Eom Y, Kang SY, Song JS, Kim YY, Kim HM. Comparison of Hoffer Q and Haigis formulae for intraocular lens power calculation according to the anterior chamber depth in short eyes. Am J Ophthalmol. 2014; 157(4):818-24. e812

8. Covert DJ, Henry CR, Koenig SB. Intraocular lens power selection in the second eye of patients undergoing bilateral, sequential cataract extraction. Ophthalmology. 2010;117(1):49-54

9. Olsen $\mathrm{T}$. Use of fellow eye data in the calculation of intraocular lens power for the second eye. Ophthalmology. 2011;118(9):1710-5.

10. Jivrajka RV, Shammas MC, Shammas HJ. Improving the second-eye refractive error in patients undergoing bilateral sequential cataract surgery. Ophthalmology. 2012;119(6):1097-101.

11. Hill W: IOL power calculations physician downloads. Haigis forrmula optimization. Available at: http://doctor-hill.com/physicians/download.htm. Accessed 11 June 2015.

12. Li Y, Bao FJ. Interocular symmetry analysis of bilateral eyes. J Med Eng Technol. 2014;38(4):179-87.

13. Jabbour J, Irwig L, Macaskill P, Hennessy MP. Intraocular lens power in bilateral cataract surgery: whether adjusting for error of predicted refraction in the first eye improves prediction in the second eye. J Cataract Refract Surg. 2006;32(12):2091-7.

14. Sheard RM, Smith GT, Cooke DL. Improving the prediction accuracy of the SRK/T formula: the T2 formula. J Cataract Refract Surg. 2010;36(11):1829-34.

15. Eom Y, Kang SY, Song JS, Kim HM. Comparison of the actual amount of axial movement of 3 aspheric intraocular lenses using anterior segment optical coherence tomography. J Cataract Refract Surg. 2013;39(10):1528-33.
Submit your next manuscript to BioMed Central and we will help you at every step:

- We accept pre-submission inquiries

- Our selector tool helps you to find the most relevant journal

- We provide round the clock customer support

- Convenient online submission

- Thorough peer review

- Inclusion in PubMed and all major indexing services

- Maximum visibility for your research

Submit your manuscript at www.biomedcentral.com/submit
Biomed Central 